\title{
ANALYSIS OF WELDED POLYPROPYLENE STRUCTURES: COMBINATION OF EXPERIMENTS AND SIMULATIONS
}

\author{
Theresa Distlbacher*, Martin Reiter, Zoltan Major
}

\author{
Johannes Kepler University, Faculty of Engineering and Natural Sciences, Institute of Polymer Product \\ Engineering, Altenbergerstraße 69, 4040 Linz, Austria \\ * corresponding author: theresa.distlbacher@jku.at
}

Abstract. The effect of joining by extrusion welding on the tensional stiffness and strength of a Polypropylene copolymer was analysed. Short-term and creep tests with laboratory specimens were conducted. Welded joint sub-components were simulated with the finite element method and the results were validated by experiments.

KEYwORDS: thermoplastic welding, extrusion-welding, FEM, welded structure.

\section{INTRODUCTION}

Extrusion welding is a joining technology suitable for manufacturing of large and complex polymeric structures. Filler material is plasticised by a small extruder and the welding partners are pre-heated locally to soften the parent material in the joining area. The molten filler then is deposited in the weld gap between the joining partners. Consolidation and solidification of the melt gives the joint its strength. [1]

This technology is well established in the industry but there is hardly any information available about the impacts of this process on the material properties and the mechanical behaviour of welded components. The presented work examined conceivable altering of the material in terms of true stress true strain relation. Also possible implication to the joint geometry was determined by analysing stiffness and strength of subcomponents based on Finite Element Method (FEM) simulations combined with experimental tests.

\section{MATERIAL}

Under examination was a semi-crystalline Polypropylene copolymer with enhanced low temperatures impact resistance. Mechanical short-term tension tests were performed and elastic-plastic material models for the FEM based on the experimental results were developed.

\subsection{Material Testing}

Two types of cylindrically laboratory specimens with a diameter of $8 \mathrm{~mm}$ and a gauge length of $30 \mathrm{~mm}$ were prepared. Figure 2 a shows drawings of the laboratory specimen types, the reference specimen type (a.1), without any weld and the weld specimen (a.2) containing an extrusion K-weld. Short-term uni-axial tensile tests at ambient temperature were performed with a tension rate of $1 \mathrm{~mm} / \mathrm{s}$. Figure 2-b illustrates the longitudinal strain on the surface of the specimen measured with a digital image correlation system during testing. A concentration of strain can be observed at the location of the K-weld. Evaluations of strains in longitudinal and transversal direction were done locally in those areas highlighted in Figure2 2 $\mathrm{b}$ by a rectangle. In further data processing steps a logarithmic measure for true strain was used.

$$
\varepsilon(t)=\ln \left(1+\epsilon_{l}(t)\right)
$$

True stress is related to the actual minimum cross section of the specimen and was calculated according to the following equation.

$$
\sigma(t)=\frac{F(t)}{A(t)}=\frac{F(t)}{\epsilon_{t}(t)^{2} A_{0}}
$$

Current values for the cross section can be derived by multiplying the initial cross section with the squared current transversal strain.

\subsection{Material Modelling}

From the true stress-strain relations, the constitutive parameters for an isotropic multi-linear elastic-plastic material model were determined.

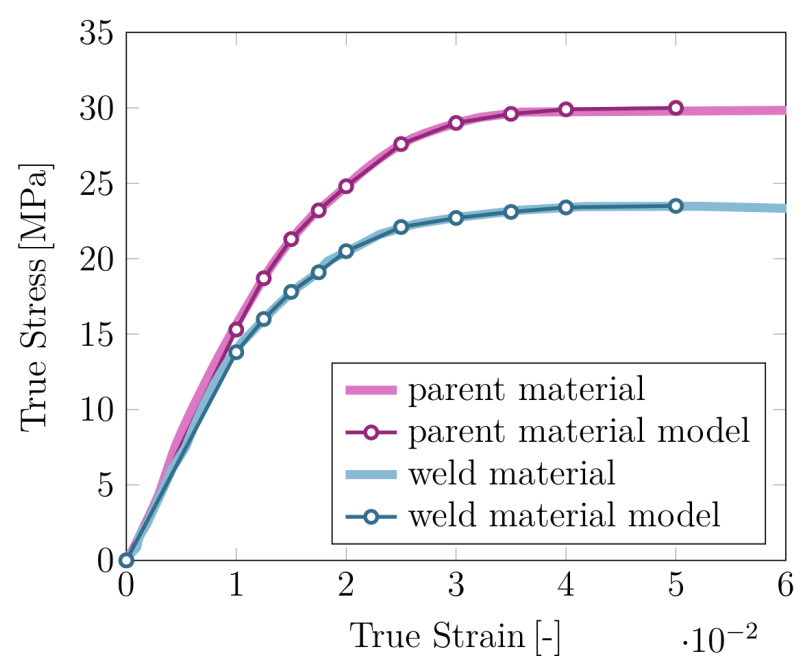

FIGURE 1. Experimental results from tensile tests with parent and weld materials and the resulting material models. 
(a.1)

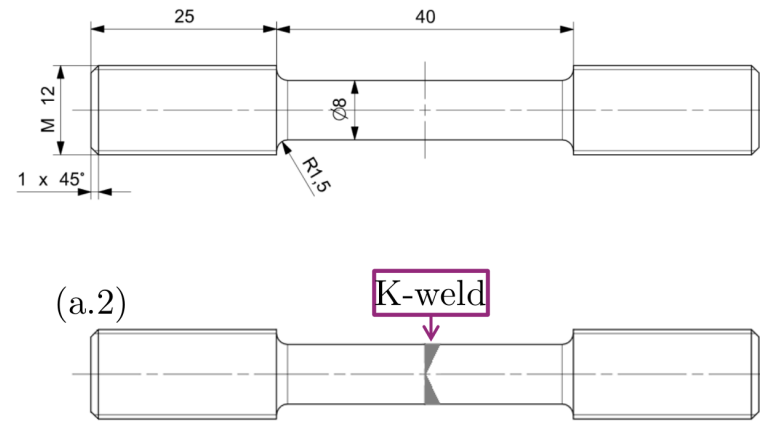

(b)

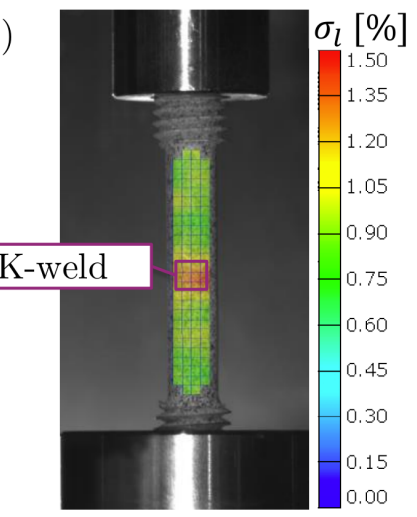

FigURE 2. Drawing of laboratory specimens: without weld (a.1), with K-weld (a.2); longitudinal strain measured during testing (b).

An illustration of the experimentally determined true stress over true strain and the material model curves for parent material and extrusion K-weld can be seen in Figure 1. This graph outlines a reduction in stiffness as well as strength of the welded material compared to the parent material. The elastic-plastic material model is an appropriate representation of the polymeric material's behaviour, even though the model is primarily applied for a large variety of metals. Polymeric materials exhibit not only elastic and plastic but also viscous behaviour $([2,3])$. Viscoelasticity is not included in this simple model. Therefore, an arbitrary chosen apparent Young's modulus and a yield point were defined for the FE software NX Nastran (Siemens PLM Software Inc./USA).

These apparent values should not be confused with the actual, physical ones resulting from tension tests. Table 1 lists the physical and apparent mechanical behaviours of the parent material.

\begin{tabular}{llcc}
\hline & & physical & apparent \\
\hline $\mathrm{E}$ & {$[\mathrm{MPa}]$} & 1534 & 1530 \\
\hline$\sigma_{y}$ & {$[\mathrm{MPa}]$} & 29.9 & 15.3 \\
\hline$\epsilon_{y}$ & {$[-]$} & 0.04 & 0.01 \\
\hline
\end{tabular}

TABLE 1. Physical and apparent parent material properties.

Basic models like this are convenient in use and could be capable also for representing some polymeric materials in special cases. In this work it was used because no unloading or dynamic effects were simulated. The application furthermore requires the analysis of time dependent behaviour (relaxation, creep, rate dependency), this simple model is not applicable and advanced models have to be utilized.

\section{Subcomponent}

The extrusion welding technology is not only used for butt-joints but more often to manufacture more complex joint-geometries like T-joints. Therefore, weld samples were manufactured according to the standard of the German Welding Society (DVS [4]). A T-joint

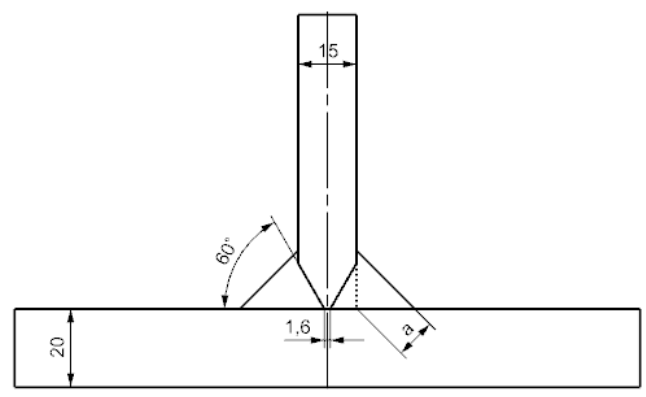

FiguRE 3. T-joint geometry.

geometry as shown in Figure 3 was analysed. One meter long weld samples were made by joining a $20 \mathrm{~mm}$ and a $15 \mathrm{~mm}$ thick extruded Polypropylene copolymer sheet via extrusion welding technology. Then, subcomponents with a length of $60 \mathrm{~mm}$ were cut off these weld samples.

\subsection{Subcomponent Testing}

The experiments were conducted with the servohydraulic testing system MTS 852 Damper Test System (MTS Systems Corporation, Eden Prairie, USA).

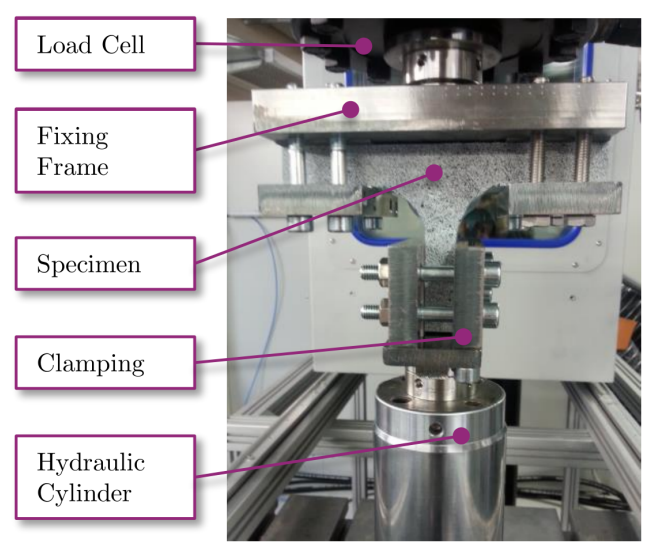

Figure 4. Subcomponent test setup. 


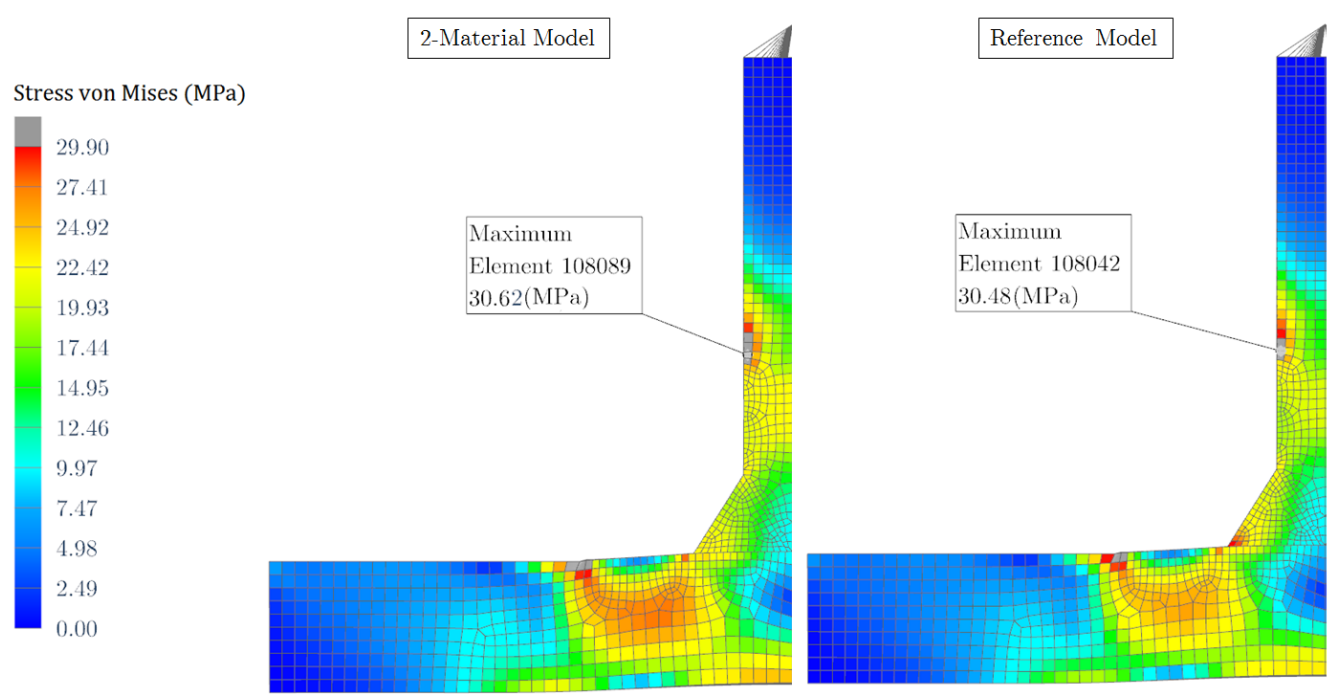

FiguRE 5. FE stress results of the 2-Material (left) and the reference model (right).

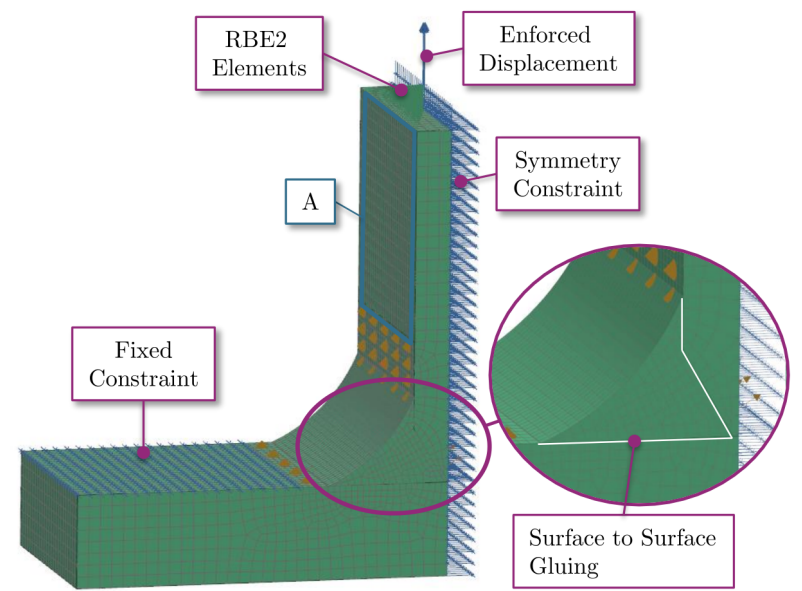

Figure 6. FE model of the T-joint.

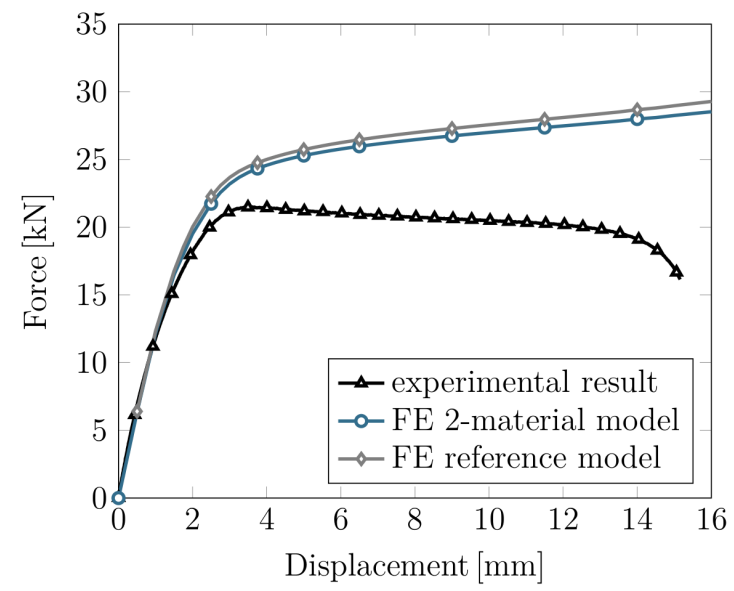

Figure 7. Experimental and FE analysis results.

degrees of freedom.

The parameters of the material models described in Section 2.1 were assigned to the mesh. Two different simulation sub-cases were created:

- 2-material model: The K-weld material model was assigned to the weld geometries and the parent material model was assigned to the sheets.

- Reference model: The parent material model was assigned to the sheet and the weld geometries.

The sub-cases are identically in terms of mesh, boundary conditions and applied load. Also the same solver, the NX Nastran's solution type SOL106, a basic nonlinear implicit solver was used.

\subsection{Results and Validation}

In Figure 7 the measured force is plotted against displacement of the plunger for the experiment, the 2-material FE model and the FE reference model.

One can see a nearly linear increase in force until a displacement of $1.5 \mathrm{~mm}$. The simulation fits the experiment very well in this region. Above a force nent was fixed by a boundary condition locking all 


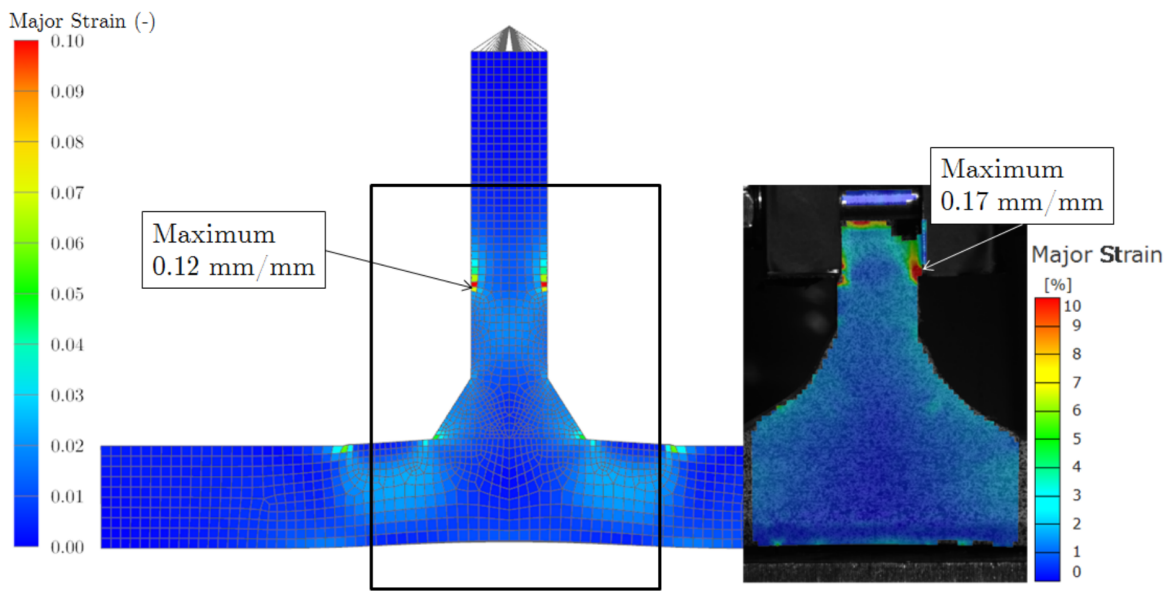

FiguRE 8. Local validation of the subcomponent reference model FE Analysis at an actuator displacement of $2 \mathrm{~mm}$.

of $15 \mathrm{kN}$ the curves level off until they reach another nearly linear region. The experimentally evaluated curve shows a negative slope and the simulated ones still exhibit a slight upwards trend. Difference between experiment and simulation in the force-displacement graph might be caused by the ideal representation of the fixing in the simulation. The FE results of the 2-material model and the reference model are nearly equal.

Evaluation of the stress distribution on the subcomponent's surface was done to find out possible local effects provoked by the second material model. In Figure 5 the stress von Mises at a displacement of $2 \mathrm{~mm}$ of the 2-material model is compared to those of the reference model. Elements where the stress has reached the physical yield stress of $29.9 \mathrm{MPa}$ are visualized in grey. Yield is already reached in those regions where the fixing constraint starts. In general one cannot see a significant differences between 2-material model and reference model in the stress distribution. Only a slightly reduced stress in the lower weld toe can be observed for the 2-material model, which is a result of the more flexible weld material.

Locally the simulation results were validated by comparing the measured major strain at the surface of the subcomponent with the simulated one, see Figure 8 The experimentally measured and the simulated maximum strain was found at the same location, where the clamping of the $15 \mathrm{~mm}$ thick sheet starts. A maximum strain of 0.17 was measured and the simulated strain was $30 \%$ smaller.

\section{Conclusions}

This work has shown that with validated data a material model for extrusion welded structures can be easily generated, to analyse larger welded structures by FEM. The elaborated material models take a nonlinear stress-strain behaviour of parent and weld material into account. A significant change in stiffness and strength of the examined material resulting from the extrusion welding process was found.
Due to the nature of extrusion welding process imperfections in the weld seam and interface are difficult to avoid. These irregularities and voids are crucial for the structural integrity. Considering such effects in FE Analysis would allow further structural improvements and reliable lifetime predictions. In future works the focus will be given to fracture mechanical analyses, including the crack propagation in the extrusion weld.

\section{LisT OF SYMBOLS \\ $A$ Cross section \\ $A_{\mathrm{o}}$ Initial cross section \\ $E$ Young's modulus \\ $F \quad$ Force \\ $\epsilon$ True strain \\ $\epsilon_{l} \quad$ Longitudinal strain \\ $\epsilon_{t} \quad$ Transversal strain \\ $\epsilon_{y} \quad$ Strain at yield \\ $\sigma$ True stress \\ $\sigma_{y} \quad$ Yield stress}

\section{ACKNOWLEDGEMENTS}

This work is part of a Master Thesis carried out at the Institute of Polymer Product Engineering (Johannes Kepler University, Linz-Austria) in cooperation with the Rosenbauer AG (Leonding-Austria). The author gratefully thanks the institute and the company for initiation of this research topic and for the scientific as well as the financial support.

\section{REFERENCES}

[1] D. A. Grewell, A. Benatar, J. B. Park. Plastics and composites welding handbook. Carl Hanser Verlag, Munich, 2003.

[2] R. S. Lakes. Viscoelastic Materials. Cambridge University Press, 2009.

[3] Y.-H. Lin. Polymer Viscoelasticity: Basics, Molecular Theories, Experiments and Simulations. World Scientific Publishing Company, New Jersey, 2nd edn., 2010.

[4] Fügen von Kunststoffen - Taschenbuch DVS Merkblätter und Richtlinien. DVS-Verlag, Düsseldorf, 13th edn., 2012. 